\title{
A MEMÓRIA ORGANIZACIONAL NOS PROCESSOS DE GESTÃO DO CONHECIMENTO: UM ESTUDO NA UNIVERSIDADE FEDERAL DO PARANÁ
}

\author{
LA MEMORIA ORGANIZACIONAL EN LOS PROCESOS \\ DE LA GESTIÓN DEL CONOCIMIENTO: UN ESTUDIO EN \\ LA UNIVERSIDAD FEDERAL DEL PARANÁ
}

\author{
Maria Olívia Ferreira Pereira* \\ Helena de Fátima Nunes Silva** \\ José Simão de Paula Pinto ${ }^{\star * *}$
}

\begin{abstract}
RESUMO
Introdução: A Memória Organizacional permite a identificação, o registro, o uso e reuso de informações e conhecimentos construídos a partir da prática. As Universidades precisam aperfeiçoar as suas práticas relacionadas à utilização e reutilização de informações decorrentes de experiências vivenciadas e aprendidas por elas.
\end{abstract}

Objetivo: Busca identificar a memória organizacional como um elemento que envolve a representação do conhecimento e da informação como uma prática da Gestão do Conhecimento na Universidade Federal do Paraná.

Metodologia: Estudo de natureza exploratória, apoiado em uma revisão da literatura. A coleta de dados foi realizada por meio de um questionário online com os funcionários que trabalham na Pró-Reitoria de Pesquisa e PósGraduação - PRPPG da UFPR.

Resultados: Os resultados apresentam dados sobre a escolaridade do pessoal estudado. É observado que os funcionários percebem a importância da Memória Organizacional, mas ainda não tem consciência da sua relevância como elemento essencial de gestão.

Conclusões: Conclui que a memória organizacional deve ser utilizada como um instrumento que propicia o compartilhamento e reuso do conhecimento corporativo e individual.

\footnotetext{
* Mestrado em Ciência, Gestão e Tecnologia da Informação pela Universidade Federal do Paraná, (UFPR). Técnico em Assuntos Educacionais da UFPR. E-mail:

mariaoliviafp@gmail.com

** Doutora em Engenharia de Produção pela Universidade Federal de Santa Catarina (UFSC). Professora do Curso de Gestão da Informação da Universidade Federal do Paraná (UFPR). E-mail: helenanuness@gmail.com

*** Doutor em Medicina pela Universidade Federal do Paraná (UFPR). Professora da UFPR. E-mail: simao@ufpr.br
} 
Maria Olívia Ferreira Pereira, Helena de Fátima Nunes Silva, José Simão de

Paula Pinto

A memória organizacional nos processos de gestão do conhecimento: um estudo na Universidade Federal do Paraná

Palavras-chave: Gestão do conhecimento. Memória organizacional. Compartilhamento do conhecimento. Reuso do conhecimento. Cultura organizacional.

\section{INTRODUÇÃO}

Na sociedade da informação, informação e conhecimento são riquezas que geram riquezas. Assim, competências ligadas ao saber, ao saber-fazer e ao saber-agir relacionados à informação e ao conhecimento são fundamentais para que os profissionais garantam sua empregabilidade e as organizações assegurem sua sobrevivência.

A capacidade produtiva não é mais totalmente dependente de capital e de equipamentos. Os ativos de informação e de conhecimento tornam-se cada vez mais importantes. Na economia da informação, o sucesso é resultado da mobilização dos ativos da informação e do conhecimento de uma comunidade e do apoio às empresas para enfrentar um novo ambiente de negócios (BATISTA, 2012).

Desta forma, a informação é reconhecidamente incomensurável e coloca-se cada vez mais o problema da seleção daquela que é pertinente para o trabalho específico, pois a disponibilidade de atenção de cada um é limitada. A questão não é a falta de informação, mas o estabelecimento adequado dos processos de coleta, tratamento, disseminação das informações corretas e relevantes para a solução de problemas organizacionais

As Universidades inseridas nesse contexto também precisam aperfeiçoar as suas práticas relacionadas à utilização e reutilização de informações decorrentes de experiências vivenciadas e aprendidas por elas. A Memória Organizacional possibilita a identificação, o registro, o uso e reuso de informações e conhecimentos construídos a partir da prática.

Em qualquer organização, e ainda mais naquelas intensivas em conhecimento, a Memória Organizacional - MO - deve ser efetiva, para 
Maria Olívia Ferreira Pereira, Helena de Fátima Nunes Silva, José Simão de

Paula Pinto

A memória organizacional nos processos de gestão do conhecimento: um estudo na Universidade Federal do Paraná

contribuir com o aprendizado. Para Shen e Liu (2010), as universidades são organizações intensivas em conhecimento. Sendo assim, toda universidade deve considerar a importância da $\mathrm{MO}$, pois ela permite a capitalização, distribuição, utilização e troca de conhecimentos e recursos (LEBLANC; ABEL, 2007).

O propósito deste artigo é analisar os processos que integram a Memória Organizacional e a sua influência como elemento de aprendizagem na Pró-Reitoria de Pesquisa e Pós-Graduação da Universidade Federal do Paraná (UFPR). Sob esse enfoque, a informação assume um papel de destaque, passando a se constituir em um dos recursos para a sobrevivência e desenvolvimento de uma organização.

Nessa perspectiva, a MO é uma representação explícita e persistente do conhecimento e das informações críticas para uma organização, cuja finalidade é facilitar o acesso, compartilhamento e reuso entre os membros da organização. Na visão de Freire et al. (2012, p. 44) "[...] o conhecimento é inerente às pessoas que formam a organização e [...] um modelo de memória organizacional deve ser estruturado para gerir a interação do indivíduo com o grupo e registrar todos os processos."

As memórias mantidas por uma organização constituem um mapa do seu passado que contém grande volume de informação. Duncan e Weiss (1979 apud MORESI, 2006) defendem que o conhecimento organizacional é essencial ao aprendizado e adaptação organizacionais. Portanto, a informação armazenada deve ser útil e atender a necessidades importantes na organização, facilitando a recuperação de conhecimento relevante que agrega valor aos seus objetivos estratégicos.

Para reusar a memória, o usuário precisa recontextualizar a informação, transportá-la para a nova situação. A informação, caso não seja de uso do próprio indivíduo que a gerou, precisa ser compreendida 
Maria Olívia Ferreira Pereira, Helena de Fátima Nunes Silva, José Simão de

Paula Pinto

A memória organizacional nos processos de gestão do conhecimento: um estudo na Universidade Federal do Paraná

no propósito de uso do novo usuário (ACKERMAN; HARVERSON, 1998). Nessa linha de pensamento, uma MO que contenha apenas $O$ conhecimento formal limita 0 processo de recontextualização e adequação para utilização no momento presente.

Segundo Argote e Miron-Spektor (2011), a MO se preocupa com a reutilização e compartilhamento do conhecimento para utilizá-lo nas atividades atuais, melhorando assim sua eficácia organizacional.

\section{REFERENCIAL TEÓRICO}

A Gestão do Conhecimento (GC) é entendida como "a arte de gerar valor a partir de bens intangíveis da organização" (SVEIBY, 1998, p. 1). O tema centra-se em aproveitar recursos existentes na organização, proporcionando a seus integrantes o emprego de melhores práticas e a reinvenção de processos (SVEIBY, 1998).

A GC está, conforme Fleury e Fleury (2000), imbricada nos processos de aprendizagem nas organizações, conjugando três processos: (1) aquisição e desenvolvimento de conhecimentos; (2) disseminação; e (3) construção de memórias em um único processo coletivo de elaboração das competências necessárias à organização.

De acordo com Nonaka e Takeuchi (1997), o conhecimento pode ser classificado em dois tipos complementares: o explícito abrange aquilo que pode ser articulado na linguagem formal e é, portanto, de fácil transmissão, podendo ser expresso sob a forma de dados brutos, de fórmulas matemáticas, palavras ou códigos. Em contraposição, o tácito abrange o conhecimento pessoal relacionado à experiência individual, envolvendo fatores subjetivos como crenças, perspectivas e sistemas de valor, dificultando a transmissão e compartilhamento com os outros, o que torna difícil a sua captura, por estarem enraizadas profundamente em ações, experiências, emoções e ideais do indivíduo. Exatamente essa característica de mutabilidade que transforma o conhecimento em 
Maria Olívia Ferreira Pereira, Helena de Fátima Nunes Silva, José Simão de

Paula Pinto

A memória organizacional nos processos de gestão do conhecimento: um estudo na Universidade Federal do Paraná

uma poderosa ferramenta para a inovação e grande fonte de competitividade para as organizações.

O tema central da Gestão do Conhecimento é aproveitar os recursos que já existem na organização para que as pessoas busquem, acessem e utilizem as melhores práticas e as lições aprendidas em vez de gerar novamente algo que já havia sido criado. Trata de agregar valor às informações, filtrando, resumindo e sintetizando-a, dessa forma, desenvolvendo um perfil de utilização que possibilita acessar a informação necessária.

Com a Gestão do Conhecimento, busca-se estimular os profissionais a realizarem um excelente trabalho e, ao mesmo tempo, captar o conhecimento de cada um e convertê-lo em algo passível de utilização pela organização, como em novas rotinas, novas ideias e novos conceitos (VON KROGH; ICHIJO; NONAKA, 2001).

Wiig (2002) define a Gestão do Conhecimento como sendo a construção, a renovação e a aplicação deste de forma sistemática, explícita e deliberada para maximizar a efetividade organizacional e o retorno de seus ativos de conhecimento.

Seis processos servem como pilares para a GC, conforme o Quadro 1. Esses são definidos por meio da identificação de diversas atividades intimamente relacionadas (PROBST; RAUB; ROMHARDT, 2002). 
Maria Olívia Ferreira Pereira, Helena de Fátima Nunes Silva, José Simão de

Paula Pinto

A memória organizacional nos processos de gestão do conhecimento: um estudo na Universidade Federal do Paraná

\begin{tabular}{|l|l|}
\multicolumn{2}{c}{ Quadro 1 - Pilares da Gestão do Conhecimento } \\
\hline PILAR & DESCRIÇÃO \\
\hline $\begin{array}{l}\text { Identificar o } \\
\text { conhecimento }\end{array}$ & Significa analisar e descrever o ambiente da empresa. \\
\hline $\begin{array}{l}\text { Adquirir o } \\
\text { conhecimento }\end{array}$ & $\begin{array}{l}\text { Importar parte do conhecimento de fontes externas, através de } \\
\text { relacionamentos com clientes, fornecedores, concorrentes e } \\
\text { parceiros, suprindo as lacunas de conhecimento. }\end{array}$ \\
\hline $\begin{array}{l}\text { Desenvolver o } \\
\text { conhecimento }\end{array}$ & $\begin{array}{l}\text { Ocorre através de diferentes estágios, começando com a } \\
\text { completa falta de entendimento das relações causais envolvidas } \\
\text { em sua situação até o entendimento total que permite o } \\
\text { controle. Baseia-se no fato de que todos os conhecimentos } \\
\text { passam por um processo evolutivo. }\end{array}$ \\
\hline $\begin{array}{l}\text { Distribuir o } \\
\text { conhecimento }\end{array}$ & $\begin{array}{l}\text { Transferência do conhecimento por meio de trocas pessoais } \\
\text { entre indivíduos. }\end{array}$ \\
\hline $\begin{array}{l}\text { Utilizar o } \\
\text { conhecimento }\end{array}$ & $\begin{array}{l}\text { Assegurar a aplicação do conhecimento para o alcance de } \\
\text { benefícios organizacionais. }\end{array}$ \\
\hline $\begin{array}{l}\text { Reter o } \\
\text { conhecimento }\end{array}$ & Seleção, armazenamento e atualização. \\
\hline $\begin{array}{l}\text { Estabelecer } \\
\text { metas }\end{array}$ & $\begin{array}{l}\text { Estabelecer quais habilidades e conhecimentos devem ser } \\
\text { desenvolvidos e em que níveis. }\end{array}$ \\
\hline $\begin{array}{l}\text { Avaliar o } \\
\text { conhecimento }\end{array}$ & Decidir se as metas de conhecimento foram atingidas. \\
\hline
\end{tabular}

Fonte: Adaptado de Probst, Raub e Romhardt (2002).

Assim, para explorar e gerir ao máximo o conhecimento que mantém, uma organização deve acima de tudo armazená-lo e organizálo de forma adequada (AHLGREN, 2011). Nesse contexto surge o conceito de Memória Organizacional.

A MO é uma prática de Gestão do Conhecimento que, além de identificar e capturar o conhecimento crítico, gera impacto na efetividade da organização (JENNEX; OLFMAN, 2007).

Estes conhecimentos são facilmente capturados e estão diretamente ligados ao sucesso das organizações e de sua MO. A retenção desse conhecimento é uma das atividades da Gestão do Conhecimento, sendo a MO responsável pelos processos de captura, registro, compartilhamento, uso e reuso.

\subsection{Memória Organizacional}

A MO deve permitir responder as principais questões que se façam sobre a organização, seu ambiente, seus processos e produtos. Deve ainda funcionar como um serviço inteligente e ativo, capaz de 
Maria Olívia Ferreira Pereira, Helena de Fátima Nunes Silva, José Simão de

Paula Pinto

A memória organizacional nos processos de gestão do conhecimento: um estudo na Universidade Federal do Paraná

colocar à disposição de quem tenha que executar determinada tarefa, no tempo certo, as informações necessárias para o trabalho (SIMIÃO, 2009).

Existem inúmeras expressões utilizadas como sinônimo de Memória Organizacional, tais como, enterprise-wide knowledge base, group memory, group mind, collective memory, collective intelligence, corporate intelligence, shared knowledge base, corporate repository e know-how data base (LEHNER; MAIER, 2000). Entretanto, para Halbwach (apud WEXLER, 2002), a MO não tem o mesmo significado que memória coletiva, já que esta última expressa mais do que um conceito individual e não pode ser reduzido a memórias individuais, pois tem como base o conhecimento sociológico da comunidade e dos grupos de trabalho.

Stein (1995) faz uma coleta de referências que contribuem para a construção da expressão "Memória Organizacional" e busca relacionar a orientação teórica do autor, conceitos de memória, modelos de aprendizagem de acordo com Argyris-Schon (1978) e formas de retenção. Stein (1995) e Stein e Zwass (1995) atribuem atenção especial às formas de retenção do conhecimento como aspecto fundamental na constituição da MO, sintetizadas no Quadro 2. 
Maria Olívia Ferreira Pereira, Helena de Fátima Nunes Silva, José Simão de

Paula Pinto

A memória organizacional nos processos de gestão do conhecimento: um estudo na Universidade Federal do Paraná

Quadro 2 - Formas de Retenção

\begin{tabular}{|l|l|l|l|}
\hline $\begin{array}{l}\text { ORIENTAÇÃO } \\
\text { TEÓRICAA }\end{array}$ & \multicolumn{1}{|c|}{ CONCEITO } & $\begin{array}{l}\text { MODELO DE } \\
\text { APRENDIZAGEM }\end{array}$ & \multicolumn{1}{|c|}{$\begin{array}{c}\text { FORMAS DE } \\
\text { RETENÇÃO }\end{array}$} \\
\hline $\begin{array}{l}\text { Management } \\
\text { science }\end{array}$ & $\begin{array}{l}\text { Memória contida } \\
\text { em procedimentos }\end{array}$ & Single loop & $\begin{array}{l}\text { Pessoas, arquivos e } \\
\text { padrões de trabalho }\end{array}$ \\
\hline $\begin{array}{l}\text { Organizational } \\
\text { learning }\end{array}$ & $\begin{array}{l}\text { Memória como } \\
\text { consequência de } \\
\text { trabalho }\end{array}$ & $\begin{array}{l}\text { Single e double } \\
\text { loop }\end{array}$ & $\begin{array}{l}\text { Pessoas (cognitivo) } \\
\text { e cultura }\end{array}$ \\
\hline Communication & $\begin{array}{l}\text { Memória construída } \\
\text { a partir do ponto de } \\
\text { vista do observador }\end{array}$ & $\begin{array}{l}\text { Single e double } \\
\text { loop }\end{array}$ & $\begin{array}{l}\text { Pessoas } \\
\text { (comportamento) e } \\
\text { cultura }\end{array}$ \\
\hline $\begin{array}{l}\text { Teoria dos } \\
\text { sistemas }\end{array}$ & $\begin{array}{l}\text { Memória como } \\
\text { resultado } \\
\text { secundário do } \\
\text { processo de } \\
\text { aprendizado }\end{array}$ & Single loop & $\begin{array}{l}\text { Responsabilidades e } \\
\text { artefatos físicos } \\
\text { (fotos, gravações, } \\
\text { etc.) }\end{array}$ \\
\hline $\begin{array}{l}\text { Decision making } \\
\text { and } \\
\text { information } \\
\text { management }\end{array}$ & $\begin{array}{l}\text { Memória como } \\
\text { forma de aumentar } \\
\text { a troca de } \\
\text { informações }\end{array}$ & Single loop & $\begin{array}{l}\text { Pessoas } \\
\text { (conhecimento } \\
\text { pessoal) }\end{array}$ \\
\hline $\begin{array}{l}\text { Organizational } \\
\text { behavior }\end{array}$ & $\begin{array}{l}\text { Interpretação das } \\
\text { pessoas de seus } \\
\text { ambientes }\end{array}$ & $\begin{array}{l}\text { Single and double } \\
\text { loop }\end{array}$ & $\begin{array}{l}\text { Pessoas e seus } \\
\text { "mapas" }\end{array}$ \\
\hline
\end{tabular}

Fonte: Stein (1995).

A MO envolve a codificação de informações do passado por meio de representações que têm um significado para a organização de acordo com a interpretação de seus membros à luz da situação presente (STEIN, 1995).

Uma das principais funções da MO é aumentar a competitividade da organização, pelo aperfeiçoamento da forma como ela gerencia seu conhecimento (ABECKER et al., 1998). Nesse sentido, a MO não é apenas um acervo de informações, sendo considerada também uma "ferramenta" da organização para o gerenciamento de seus ativos intelectuais. Sua existência deve propiciar maior compartilhamento e reuso do conhecimento corporativo, do conhecimento individual e das lições aprendidas na execução das tarefas da organização.

Deste modo, a MO pode ser entendida como conhecimento corporativo que representa experiências prévias que são arquivadas e compartilhadas pelos usuários. Ela é constituída por um conhecimento 
Maria Olívia Ferreira Pereira, Helena de Fátima Nunes Silva, José Simão de

Paula Pinto

A memória organizacional nos processos de gestão do conhecimento: um estudo na Universidade Federal do Paraná

explícito (arquivos guardados como manuais corporativas e banco de dados) e conhecimento tácito (tais como intuição, opiniões e experiência) e abrange aspectos funcionais, técnicos e sociais de trabalho, o trabalhador e o ambiente de trabalho. A MO pode ser instituída dentro de uma organização por meio de reuniões, e-mails, transações, sistemas de relatórios, conferências, entre outras formas e servirá para apoiar a tomada de decisões em várias tarefas e em diferentes ambientes (NILAKANTA; MILLER; ZHU, 2006).

Para Fiedler e Welpe (2010), a MO é entendida como uma estrutura de repositórios, em que diferentes formas de conhecimentos são armazenados, e que a partir do qual esses mesmos conhecimentos podem ser constantemente recuperados.

\subsubsection{Componentes da Memória Organizacional}

Nilakanta, Miller e Zhu (2006), ao apresentar a estrutura de um modelo explica onde reside a $\mathrm{MO}$, quais são seus componentes e como eles funcionam no sistema geral de uma organização, basearam-se em um estudo já existente de Walsh e Ungson (1991). Neste modelo apresentado na Figura 1, o motor do conhecimento é o centro dessa estrutura, que igualmente interage e sustenta os outros componentes. 
Maria Olívia Ferreira Pereira, Helena de Fátima Nunes Silva, José Simão de

Paula Pinto

A memória organizacional nos processos de gestão do conhecimento: um estudo na Universidade Federal do Paraná

Figura 1 - Memória organizacional em um sistema do conhecimento organizacional

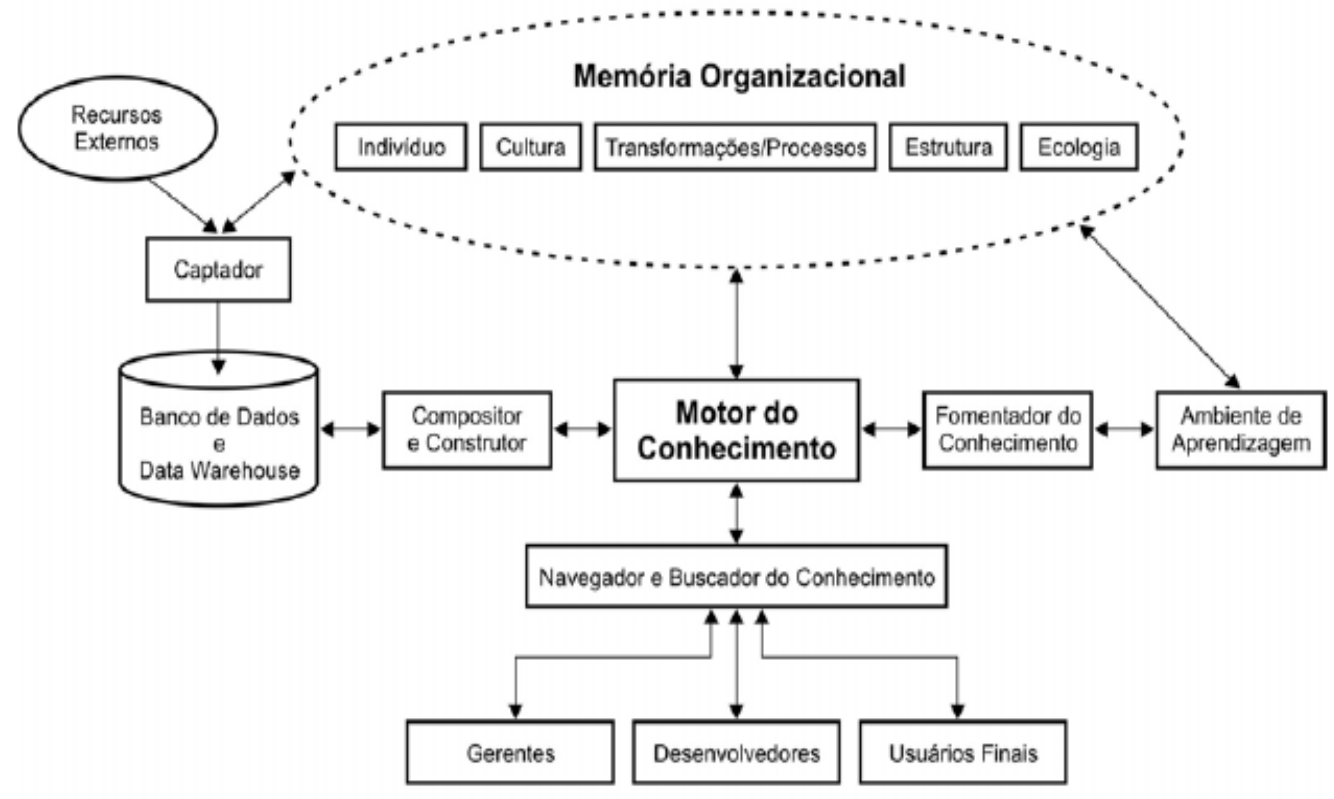

Fonte: Nilakanta, Miller e Zhu (2006).

Todos os membros da organização precisam de um acesso fácil e rápido para este repositório, em todos os níveis: operacional, tático e estratégico. Ao mesmo tempo, este modelo permite que o sistema aprenda continuamente, possibilitando assim ampliar e melhorar a MO da empresa. Nesta estrutura apresentada, é importante considerar as funções de aquisição, retenção, recuperação, integração, atualização e sua utilização (NILAKANTA; MILLER; ZHU, 2006). De acordo com Freire et al. (2012, p. 50) "são indispensáveis meios competentes de registro de informações, especialmente se relevantes."

A MO, conforme a Figura 1, é composta de cinco componentes internos e um componente externo. Os componentes internos são representados pelos indivíduos, pela cultura, pelas transformações (colaboração de grupo), pela estrutura e pela ecologia da organização. Já os arquivos impressos e digitais fazem parte do componente externo de uma organização.

Nessa perspectiva, são explicitados os componentes internos e externos de uma organização. 
Maria Olívia Ferreira Pereira, Helena de Fátima Nunes Silva, José Simão de

Paula Pinto

A memória organizacional nos processos de gestão do conhecimento: um estudo na Universidade Federal do Paraná

Indivíduos - são as pessoas que trabalham e desenvolvem as atividades adquirindo habilidades e competências que são internalizadas e processadas em suas mentes, constituindo a memória individual.

Cultura - A cultura pode ser definida como uma maneira de perceber, pensar e sentir podendo modificar-se a cada nova experiência, já que é um processo vivo. Todavia, as experiências passadas podem auxiliar decisões futuras, o que faz com que a cultura se torne um tipo de armazenagem organizacional.

Colaboração do Grupo / Transformações / Processos Informação e conhecimento são embutidos nos processos e procedimentos que acontecem diariamente nas organizações. Existe conhecimento nos processos de colocar dados em um determinado sistema e na forma de transformá-los em um produto ou em uma informação, assim como nos procedimentos padronizados, no treinamento realizado e na forma como o conhecimento dos funcionários mais experientes é repassado aos novos funcionários.

Todos os processos, colaborações ou transformações contêm conhecimento e informação, que podem ser evidentes ou escondidos. As experiências que são acumuladas ao longo do tempo tornam-se embutidas nos processos e procedimentos individuais, contudo, quando os indivíduos trabalham em conjunto o sistema utilizado pelo grupo para armazenar e processar os conhecimentos é mais complexo do que a memória individual. Assim, o trabalho em grupo exige de seus membros um sistema de memória combinado que permita ser acessado a qualquer momento (LEHNER; MAIER, 2000; WALSH; UNGSON, 1991).

Estrutura - influencia o comportamento dos indivíduos e sua relação com o ambiente. Os papéis individuais fornecem a ligação entre as memórias individuais e memória organizacional: na medida em que esses papéis se tornam padronizados ao longo do tempo, descrevendo a diferenciação de tarefas, o repositório que armazena papéis individuais é aplicável à organização. 
Maria Olívia Ferreira Pereira, Helena de Fátima Nunes Silva, José Simão de

Paula Pinto

A memória organizacional nos processos de gestão do conhecimento: um estudo na Universidade Federal do Paraná

Estrutura Física ou Ecologia do local de trabalho codifica e revela informações. A configuração física ajuda a moldar e reforçar as prescrições de comportamento dentro da organização. Dessa forma, ela retém informação sobre a organização e de seus membros.

Arquivos Externos sua importância reside no fato de que a organização não é o único repositório de seu passado. Essas fontes guardam informações sobre o seu passado e pode ser recuperadas.

O’Dell e Grayson Junior (1998) sugerem práticas que facilitam a transferência de informações que em muitos casos em que após um extenso treinamento em benchmarking das melhores práticas, foi identificado a sua prática, sugerindo outro benefício de caracterizar e compreender a abrangência desses fatores na MO. Ela é caracterizada como um sistema de conhecimento.

A representação da $\mathrm{MO}$ contempla o aprendizado e o conhecimento que influenciam o seu desenvolvimento. Esses três componentes interagem entre si resultando $\mathrm{o}$ armazenamento e a recuperação de informações relevantes para a organização na tomada de decisão e na otimização dos processos de maneira eficiente, convertendo-se em resultados eficazes.

Nessa perspectiva, a natureza dos fluxos entre os processos é examinada, considerando o tipo de conhecimento envolvido e nos fatores que podem facilitar ou inibir esse fluxo de conhecimento. Sendo que know how é essencial e, na maioria das vezes, está disponível somente na mente das pessoas. A informação valiosa está em banco de dados e documentos. Outro aspecto a ser considerado é que os erros cometidos e repetidos se devem a não consideração de experiências passadas.

Reátegui (2013) apresenta, conforme Quadro 3, a utilização de sistemas de memória organizacional (SMO's) para uso acadêmico: 
Maria Olívia Ferreira Pereira, Helena de Fátima Nunes Silva, José Simão de

Paula Pinto

A memória organizacional nos processos de gestão do conhecimento: um estudo na Universidade Federal do Paraná

Quadro 3 - Características dos Smo Baseados em Computador

\begin{tabular}{|c|c|c|c|c|c|c|}
\hline \multirow[t]{2}{*}{ SMO } & \multirow[t]{2}{*}{ Conteúdo } & \multicolumn{2}{|l|}{ Estrutura } & \multicolumn{3}{|c|}{ Processos Operacionais } \\
\hline & & Localização & Indexação & Coleta & Manutenção & Acesso \\
\hline $\begin{array}{l}\text { Repositório } \\
\text { de vídeos }\end{array}$ & \multirow[t]{8}{*}{ Explícito } & \multirow[t]{8}{*}{ Centralizada } & por computador & $\begin{array}{l}\text { contribuições, } \\
\text { filtrada }\end{array}$ & Formal & \multirow[t]{10}{*}{ Tecnológicc } \\
\hline $\begin{array}{l}\text { Repositório } \\
\text { de } \\
\text { Documentos }\end{array}$ & & & por computador & Filtrada & $\begin{array}{l}\text { Sem } \\
\text { manutenção } \\
\text { formal }\end{array}$ & \\
\hline $\begin{array}{l}\text { Repositório } \\
\text { de áudios }\end{array}$ & & & $\begin{array}{l}\text { categorizada/ } \\
\text { por computador }\end{array}$ & filtrada & $\begin{array}{l}\text { Sem } \\
\text { manutenção } \\
\text { formal }\end{array}$ & \\
\hline $\begin{array}{l}\text { Repositório } \\
\text { de recursos } \\
\text { educativos }\end{array}$ & & & $\begin{array}{l}\text { categorizada/ } \\
\text { por computador }\end{array}$ & $\begin{array}{l}\text { contribuições, } \\
\text { filtrada }\end{array}$ & $\begin{array}{l}\text { Sem } \\
\text { manutenção } \\
\text { formal }\end{array}$ & \\
\hline $\begin{array}{l}\text { COWP } \\
\text { UTPL }\end{array}$ & & & $\begin{array}{l}\text { categorizada/ } \\
\text { por computador }\end{array}$ & Filtrada & Formal & \\
\hline $\begin{array}{l}\text { Repositório } \\
\text { de } \\
\text { publicações }\end{array}$ & & & $\begin{array}{l}\text { categorizada/ } \\
\text { por computador }\end{array}$ & $\begin{array}{l}\text { contribuições, } \\
\text { filtrada }\end{array}$ & formal & \\
\hline Wik ECC & & & $\begin{array}{l}\text { categorizada/ } \\
\text { por computador }\end{array}$ & $\begin{array}{l}\text { contribuições, } \\
\text { filtrada }\end{array}$ & $\begin{array}{l}\text { sem } \\
\text { manutenção } \\
\text { formal }\end{array}$ & \\
\hline Blog ECC & & & $\begin{array}{l}\text { categorizada/ } \\
\text { por computador }\end{array}$ & $\begin{array}{l}\text { contribuições, } \\
\text { filtrada }\end{array}$ & $\begin{array}{l}\text { sem } \\
\text { manutenção } \\
\text { formal }\end{array}$ & \\
\hline slideshare & & & $\begin{array}{l}\text { categorizada/ } \\
\text { por computador }\end{array}$ & $\begin{array}{l}\text { contribuições, } \\
\text { filtrada }\end{array}$ & $\begin{array}{l}\text { sem } \\
\text { manutenção } \\
\text { formal }\end{array}$ & \\
\hline Youtube & & & $\begin{array}{l}\text { categorizada/ } \\
\text { por computador }\end{array}$ & $\begin{array}{l}\text { contribuições, } \\
\text { filtrada }\end{array}$ & $\begin{array}{l}\text { sem } \\
\text { manutenção } \\
\text { formal }\end{array}$ & \\
\hline
\end{tabular}

Fonte: Adaptado de Oliveira (2000 apud REÁTEQUI, 2013).

Inf. Inf., Londrina, v. 21, n. 1, p. 348 - 374, jan./abr. 2016.

http:www.uel.br/revistas/informacao/ 
Maria Olívia Ferreira Pereira, Helena de Fátima Nunes Silva, José Simão de

Paula Pinto

A memória organizacional nos processos de gestão do conhecimento: um estudo na Universidade Federal do Paraná

Em sua pesquisa, Reátegui (2013) mostra o envolvimento dos docentes enquanto produtores de conteúdos, e alguns aspectos informacionais, concluindo que os produtos da MO pertencem a toda a instituição e nela permanecem.

\subsubsection{Cultura Organizacional}

A cultura, segundo Zago (2005), é o elemento mais difícil de definir em uma organização, já que ela influencia todos os indivíduos em diferentes aspectos, incluindo a comunicação que facilitará ou não a transferência, o compartilhamento e a maximização do conhecimento.

Cultura pode ser entendida, como a resultante cognitiva de uma construção social que é extraída de aspectos importantes da experiência coletiva dos membros de uma sociedade que se configuram como modelos mentais, visão compartilhada ou arquétipos; os quais, uma vez incorporados, passam para o nível do inconsciente coletivo, onde muitas vezes, procedimentos não são explicáveis do porque, mas são aceitos como certos, sem questionamentos, "simplesmente" são assim. (ZAGO, 2005, p. 3).

Na perspectiva de Schein (1991, p. 247) a cultura é "o resultado dos esforços da organização para se adaptar ao ambiente e, ao mesmo tempo, conseguir a integração interna."

No processo de integração interna, os membros desenvolvem consenso sobre linguagem e categorias conceituais comuns a serem usadas para que as pessoas possam se comunicar e se entender, para que os membros possam responder ao que não conseguem entender (CHOO, 2003, p. 151)

\section{METODOLOGIA}

É um estudo exploratório desenvolvido no ambiente da Universidade Federal do Paraná na Pró-Reitoria de Pesquisa e PósGraduação. A escolha da instituição e da unidade foi por acessibilidade 
Maria Olívia Ferreira Pereira, Helena de Fátima Nunes Silva, José Simão de

Paula Pinto

A memória organizacional nos processos de gestão do conhecimento: um estudo na Universidade Federal do Paraná

e também pelas características da Unidade como produtora de pesquisa e de conhecimento.

Para a coleta de dados foi elaborado um questionário composto por questões fechadas, as quais foram respondidas utilizando-se a escala likert (1) Discordo Totalmente; (2) Discordo; (3)Concordo; (4)Concordo Totalmente. Os questionários foram enviados por e-mail para 25 funcionários e destes retornaram 22. As questões contemplaram os seguintes aspectos: caracterização do pesquisado (perfil no que se refere à escolaridade e o tempo de serviço); processos de registro do conhecimento adquirido a partir das lições aprendidas; ou seja, uso e reuso do conhecimento retirado das atividades e o tratamento das informações e conhecimentos produzidos no espaço interno. Os dados coletados foram analisados a partir das categorias pré-estabelecidas (Uso e reuso do conhecimento, tratamento das informações, conhecimentos produzidos) e, posteriormente, comparados com a literatura pertinente. Optou-se por tabular os dados conforme o número de respostas obtidas nas diferentes perguntas, categorizando-as em duas classes: Memória Organizacional (individuo) e Cultura.

\section{$4 \quad$ RESULTADOS E DISCUSSÕES}

Os resultados e análises sobre processo de Memória Organizacional da Pró-Reitoria de Pesquisa e Pós-Graduação da UFPR são apresentados a seguir. Para tanto, foram gerados gráficos os quais apresentam as percentagens relativas ao grau de escolaridade, tempo de trabalho na instituição e as percepções relativas à Memória Organizacional e Cultura.

\subsection{Perfil dos Respondentes}

Os funcionários entrevistados, conforme Gráfico 1, na sua grande maioria, ou seja, $50 \%$ tem pós-graduação, $13,64 \%$ têm ensino superior 
Maria Olívia Ferreira Pereira, Helena de Fátima Nunes Silva, José Simão de

Paula Pinto

A memória organizacional nos processos de gestão do conhecimento: um estudo na Universidade Federal do Paraná

completo, $13,64 \%$ ensino superior incompleto, totalizando $77,28 \% \mathrm{com}$ uma formação superior.

Os dados refletem um nível de escolarização alto, o qual pode agregar conhecimentos nos processos de gestão e na tomada de decisão.

Gráfico 1 - Nível de Escolaridade



Fonte: Elaborado pelos autores com base na pesquisa (2013).

No que se refere ao tempo de trabalho, o Gráfico 2 indica que $18,18 \%$ dos funcionários estão na Instituição entre três anos e cinco anos período correspondente ao estágio probatório; 9,09\% entre três e dez anos e 45\% estão há mais de dez anos. Pressupõe-se, que habilidades e competências relativas às funções estejam consolidadas, podendo assim agregar conhecimento e práticas que possibilitam o uso e reuso da informação de maneira eficiente na tomada de decisão e no gerenciamento dos processos. 
Maria Olívia Ferreira Pereira, Helena de Fátima Nunes Silva, José Simão de

Paula Pinto

A memória organizacional nos processos de gestão do conhecimento: um estudo na Universidade Federal do Paraná

Gráfico 2 - Tempo de Trabalho na Instituição

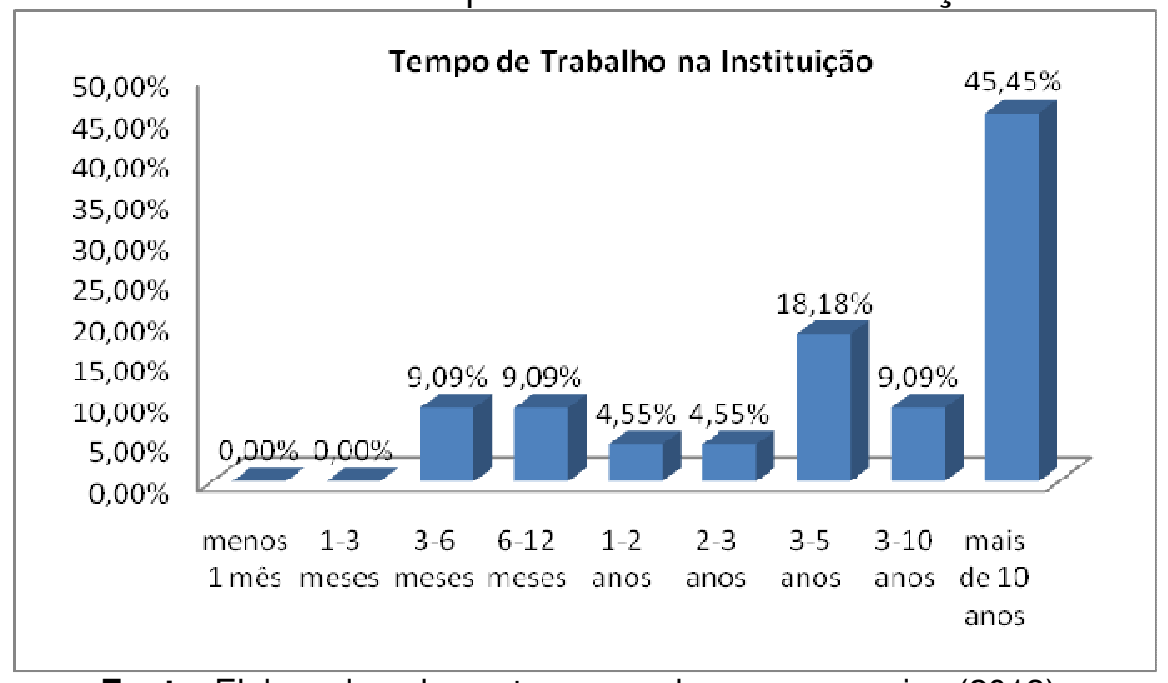

Fonte: Elaborado pelos autores com base na pesquisa (2013).

Neste sentido, o pensamento de March e Olsen (1979) ilustra os resultados encontrados quando colocam que aprender pela experiência é a escolha de alternativas com base em regras desenvolvidas a partir de um acúmulo de experiências passadas. Essa aprendizagem olha para a história passada, vai ao encontro de orientação para futuras ações. Organizações e pessoas que nelas aprendem o fazem por meio de suas interações com o ambiente - elas atuam, observam as conseqüências de suas ações, fazem inferências sobre as consequências e extraem as implicações para a ação futura.

A MO é utilizada pelas organizações como ferramenta que objetiva gerenciar os ativos intelectuais. Assim, ela não pode ser considerada somente uma ferramenta que acumula e preserva 0 conhecimento, mas sim, um instrumento que deve propiciar o compartilhamento e reuso do conhecimento corporativo e individual. A MO deve ter a capacidade de responder às principais perguntas da organização referentes ao ambiente, produtos, procedimentos e processos. Ao mesmo tempo, a MO deve funcionar como um serviço inteligente e ativo, que consegue colocar à disposição dos funcionários qualquer informação desejada no momento solicitado (LASPISA, 2007). 
Maria Olívia Ferreira Pereira, Helena de Fátima Nunes Silva, José Simão de

Paula Pinto

A memória organizacional nos processos de gestão do conhecimento: um estudo na Universidade Federal do Paraná

É de vital importância aproveitar os recursos que já existem na organização para que as pessoas busquem, acessem e utilizem as melhores práticas, as lições aprendidas em vez de gerar algo que já havia sido criado. Portanto, é necessário agregar valor às informações, filtrando, resumindo e sintetizando as informações e, dessa forma, desenvolvendo um perfil de utilização pessoal que possibilita o acesso a informação necessária

\subsection{Memória Organizacional e Cultura}

Para a análise dos resultados utilizou-se as categorias Memória Organizacional e Cultura. A Categoria Memória Organizacional engloba os processos de registro do conhecimento adquirido a partir das lições aprendidas (uso e reuso do conhecimento retirado das atividades) e o tratamento das informações e conhecimentos produzidos no espaço interno. A Cultura organizacional permeia a geração, coleta, compartilhamento, uso e reuso das informações e conhecimentos produzidos na instituição.

Pelos resultados, representados no Gráfico 3, observa-se que 46,46\% dos funcionários percebem a importância da Memória Organizacional, mas ainda não tem consciência da sua relevância como elemento essencial de gestão, utilizando-a como pano de fundo para a Gestão do Conhecimento.

Nessa perspectiva, Walsh e Ungson (1991) definem a memória organizacional como um meio pelo qual a informação do passado é aplicada em decisões presentes. Sob um aspecto mais restrito, a memória organizacional envolve a representação do conhecimento e da informação em uma organização. Ela pode incluir conhecimento sobre os objetivos, planos, produtos, processos de produção, clientes, resultados financeiros, experiências e perícia na resolução de problemas. 
Maria Olívia Ferreira Pereira, Helena de Fátima Nunes Silva, José Simão de

Paula Pinto

A memória organizacional nos processos de gestão do conhecimento: um estudo na Universidade Federal do Paraná

Essa preocupação com a preservação do conteúdo também guarda relações com a recuperação do conhecimento ou informação da MO. Para reusar a memória, o usuário precisa recontextualizar a informação, transportá-la para a nova situação.

Gráfico 3 - Memória Organizacional e Cultura

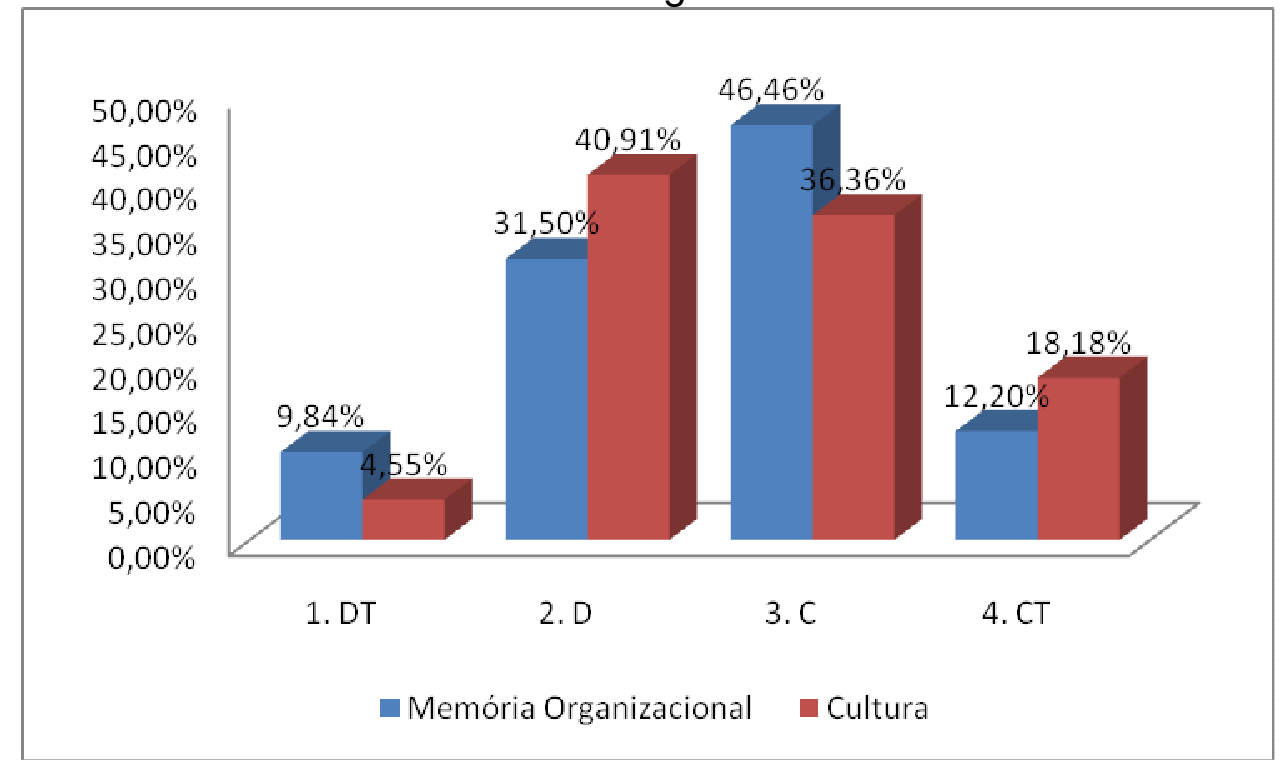

Fonte: Elaborado pelos autores com base na pesquisa (2013).

\section{Legenda:}

(1) DT - Discordo Totalmente

(2) D - Discordo

(3) C - Concordo

(4) CT - Concordo Totalmente

Outro aspecto levantado é a cultura em que $54,54 \%$ percebem a sua relevância, pois ela permeia a missão institucional e deve incentivar os indivíduos a transferirem informações, compartilharem e inovarem seus conhecimentos.

Os dados refletem a sua importância, mas seria necessária uma pesquisa mais aprofundada, pois é questionável se realmente há uma cultura voltada à memória organizacional e se os funcionários a utilizam nos processos internos no sentido de alavancar estratégias, possibilitando a interação, os valores e os comportamentos dos indivíduos no desenvolvimento de habilidades e competências. 
Maria Olívia Ferreira Pereira, Helena de Fátima Nunes Silva, José Simão de

Paula Pinto

A memória organizacional nos processos de gestão do conhecimento: um estudo na Universidade Federal do Paraná

Nesse aspecto, a cultura é apontada como uma das principais razões de sucesso de empresas e instituições. Sendo vista como uma forma de obter eficácia, eficiência e efetividade nas ações de uma organização.

Para Costa (2011, p. 223) a cultura organizacional de uma empresa ou instituição pode atender ou não às demandas de seus clientes. Quando atende, fortalece-se a estrutura de poder interna, ratificando-se as cadeias hierárquicas e os processos decisórios e operativos. Quando não, instala-se a crise que, para ser superada, exige crítica ampla e profunda a respeito daqueles processos e abala as cadeias hierárquicas e seus círculos de poder. A situação ideal de uma empresa ou instituição é aquela na qual ela está operando de maneira consciente, não somente em relação ao ambiente externo, mas também quanto à sua cultura organizacional, que pode ser entendida como sua capacidade de correlacionar respostas às demandas externas e internas de poder.

Os obstáculos à construção de sistemas de memória organizacional perpassam não só pela categoria técnica, mas também pela cultural. Conklin (2001) chama à atenção para algumas questões a serem levadas em consideração:

a) como tornar o processo de captura do conhecimento fácil ou mesmo transparente;

b) como fazer a recuperação e reuso fácil ou transparente;

c) como assegurar relevância e inteligibilidade do conhecimento recuperado.

Possivelmente, o maior obstáculo para o sucesso da MO seja a mudança cultural. Para atingir o objetivo de preservação dos ativos de conhecimento, faz-se necessário rever as práticas da organização, de sua força de trabalho e de seus costumes, tornando possível o processo de mudança e possibilitando a criação de novas ferramentas e novas práticas (CONKLIN, 2001).

A MO pode armazenar os mais diversos recursos com os quais a organização conta durante seu ciclo de vida, o que implica a 
Maria Olívia Ferreira Pereira, Helena de Fátima Nunes Silva, José Simão de

Paula Pinto

A memória organizacional nos processos de gestão do conhecimento: um estudo na Universidade Federal do Paraná

possibilidade de construção de memórias diferenciadas de acordo com a necessidade de cada organização. A expertise coletiva e experiência cultivada por meio das redes humanas podem ser extraídas, armazenadas e utilizadas para a melhoria do desempenho organizacional, permitindo às organizações "saber o que elas sabem" (O'DELL apud ALWIS; HLUPIC; RZEVSKI, 2004), sendo um instrumento para geração de ideias e compreensão sobre sua própria experiência, gerando conhecimentos adicionais e servindo como diferencial.

Desta forma, os processos de registro do conhecimento das lições aprendidas e o tratamento das informações e conhecimentos produzidos no espaço interno são fundamentais para que seja efetivada a $\mathrm{MO}$ a qual depende de uma Cultura Organizacional propícia ao uso e reuso do conhecimento retirado das atividades e experiências das pessoas.

\section{CONSIDERAÇÕES FINAIS}

Este estudo pautou-se em rever o pensamento de diversos autores que desenvolvem trabalhos com foco na Memória Organizacional objetivando a análise de dados coletados em uma Instituição de Ensino Superior. A análise realizada foi sob a perspectiva de Gandon (2002, p. 28)

Uma memória organizacional é uma representação persistente, explicita, não incorporada; um índice do conhecimento e da informação, ou de suas fontes, em uma organização, de forma a facilitar o acesso, o compartilhamento e a reutilização (do conhecimento, da informação e suas fontes) pelos membros da organização, em suas atividades individuais e coletivas (GANDON, 2002, p. 28).

Constatou-se que a memória e a cultura estão presentes nos processos de gestão, mas não estão explicitados nas rotinas e não são utilizados de maneira eficiente, impossibilitando estratégias que agreguem informações na tomada de decisão. Acredita-se que tanto a memória organizacional como a cultura precisam ser trabalhados na 
Maria Olívia Ferreira Pereira, Helena de Fátima Nunes Silva, José Simão de

Paula Pinto

A memória organizacional nos processos de gestão do conhecimento: um estudo na Universidade Federal do Paraná

abordagem proveniente da gestão do conhecimento, em suas interfaces com o aprendizado organizacional.

Nessa perspectiva, o desenvolvimento da memória está diretamente relacionado à aprendizagem organizacional e a gestão do conhecimento.

A representação da memória organizacional contempla o aprendizado e o conhecimento que influenciam o seu desenvolvimento. Esses três componentes interagem entre si resultando 0 armazenamento e a recuperação de informações relevantes para a organização na tomada de decisão e na otimização dos processos convertendo-se em resultados eficazes.

Nesse sentido, este estudo exploratório, explicitou alguns conceitos sobre a memória organizacional e que podem contribuir com a gestão das organizações que os adotam, bem como para a implementação de ações relacionadas à gestão do conhecimento.

Pela característica do estudo, os resultados são limitados à instituição estudada. Acredita-se que a ampliação da pesquisa em outras universidades deve contribuir para o entendimento de como são percebidos e utilizados na prática, os conceitos de Memória e Cultura Organizacional.

\section{REFERÊNCIAS}

ABECKER, Andreas et al. Towards a technology for organisational memories. IEEE Intelligent Systems, Los Alamitos, v. 13, n. 3, p. 40-48, may/jun. 1998.

ACKERMAN, Mark S.; HALVERSON, Christine. Considering an organization's memory. In: ACM CONFERENCE ON COMPUTER SUPPORTED COOPERATIVE WORK, 1998, Seattle. Proceedings... Washington: ACM, 1998. p. 39-48. 
Maria Olívia Ferreira Pereira, Helena de Fátima Nunes Silva, José Simão de

Paula Pinto

A memória organizacional nos processos de gestão do conhecimento: um estudo na Universidade Federal do Paraná

AHLGREN, Riikka. Software patterns, organizational learning and software process improvement. Jyväskylä: Faculty of Information Technology, University of Jyväskylä, 2011.

ALWIS, Dee; HLUPIC, Vlatka; RZEVSKI, George. Designing organizational memory in knowledge intensive companies: a case study. 2004. Disponível em:

<http://www.km.fhkoeln.de/content/dateien/Publikationem/Rzevski _07_Organizational Memory KM Book.pdf>. Acesso em: 12 out. 2012.

ARGOTE, Linda; MIRON-SPEKTOR, Ella. Organizational learning: from experience to knowledge. Organization Science, Providence, v. 22, n. 5, p. 1123-1137, 2011.

ARGYRIS, Chris; SHÖN, Donald A. Organizational learning: a theory of action perspective. Massachusetts: Addison-Wesley, 1978.

BATISTA, Fábio Ferreira. Modelo de gestão do conhecimento para a administração pública brasileira: como implementar a gestão do conhecimento para produzir resultados em benefício do cidadão. Brasília: Ipea, 2012.

CHOO, Chun Wei. A organização do conhecimento: como as organizações usam a informação para criar significado, construir conhecimento e tomar decisões. São Paulo: Senac, 2003.

CONKLIN, Jeff. Designing organizational memory: preserving intellectual assets in a knowledge economy. 2001. Disponível em: $<$ http://cognexus.org/dom.pdf>. Acesso em: 25 jun. 2012.

COSTA, Sérgio Paulo Muniz. Cultura organizacional nas empresas e nas instituições. In: TAPARANOFF, Kira (Org.). Aprendizado organizacional: fundamentos e abordagens multidisciplinares. Curitiba: lbpex, 2011. v. 1. Cap. 7.

FIEDLER, Marina; WELPE, Isabell. How do organizations remember? The influence of organizational structure on organizational memory. Organization Studies, Berlin, v. 31, n. 4, p. 381-407, 2010.

FLEURY, Afonso Carlos Correa; FLEURY, Maria Teresa Leme. Estratégias empresariais e formação de competências: um quebracabeça caleidoscópio de uma indústria brasileira. São Paulo: Atlas, 2000.

FREIRE, Patrícia Sá et al. Memória organizacional e seu papel na gestão do conhecimento. Revista de Ciências da Administração, Florianópolis, v. 14, n. 33, p. 41-51, ago. 2012. 
Maria Olívia Ferreira Pereira, Helena de Fátima Nunes Silva, José Simão de

Paula Pinto

A memória organizacional nos processos de gestão do conhecimento: um estudo na Universidade Federal do Paraná

GANDON, Fabien. Distributed artificial intelligence and knowledge management: ontologies and multi-agent systems for a corporate semantic web. 2002. $483 \mathrm{f}$. Thesis (Scientific philosopher doctorate in informatics) - Doctoral school of sciences and technologies of information and communication, INRIA and University of Nice Sophia Antipolis, Nice, 2002.

JENNEX, Murray E.; OLFMAN, Lorne. Organizational memory. In: HOLSAPPLE, Clyde W. Handbook on knowledge management. Kentucky: Springer Verleg, 2004. p. 207-234.

LASPISA, David Frederick. Influência do conhecimento individual na memória organizacional: um estudo de caso em call centers. 2007. 120 f. Dissertação (Mestrado em Engenharia e Gestão do Conhecimento) - Universidade Federal de Santa Catarina, Florianópolis. 2007.

LEBLANC, Adeline; ABEL, Marie-Hélène. Using organizational memory and forum in an organizational learning context. 2007. Disponível em: <http://ieeexplore.ieee.org/stamp/stamp.jsp?tp=\&arnumber=4444234>. Acesso em: $18 \mathrm{dez} .2013$.

LEHNER, Franz; MAIER, Ronald K. How can organizational memory theories contribute to organizational memory systems? Information Systems Frontiers, v. 2, n. 3/4, p. 277- 298, 2000. Disponível em: $<$ https://www.researchgate.net/publication/ 220199019_How_Can_Organizational_Memory_Theories_Contribute_to _Organizational_Memory_Systems>. Acesso em: $18 \mathrm{dez} . \overline{2} 013$.

MARCH, James G.; OLSEN, Johan. P. Organizational learning and the ambiguity of the past. In: (Ed.). Ambiguity and choice in organizations. Oslo: Universitetsforlaget, 1979. p. 54-68.

MORESI, Eduardo A. D. Perspectivas de pesquisa em inteligência organizacional. In: CONGRESSO IBEROAMERICANO DE GESTÃO DO CONHECIMENTO E INTELIGÊNCIA COMPETITIVA - GeCIC, 2006, Curitiba. Anais... Curitiba, 2006. Disponível em:

<http://www.gecic.com.br/artigos.asp?07>. Acesso em: 24 nov. 2012.

NILAKANTA, Sree; MILLER, L. L.; ZHU, Dan. Organizational memory management: technological and research issues. Journal of Database Management, Pennsylvania, v. 17, n. 1, p. 85-94, jan./mar. 2006.

NONAKA, Ikujiro; TAKEUCHI, Hirotaka. Criação de conhecimento na empresa: como as empresas japonesas geram a dinâmica da inovação. 9. ed. Rio de Janeiro: Campus, 1997. 
Maria Olívia Ferreira Pereira, Helena de Fátima Nunes Silva, José Simão de

Paula Pinto

A memória organizacional nos processos de gestão do conhecimento: um estudo na Universidade Federal do Paraná

O'DELL, Carla; GRAYSON JUNIOR, Jackson. If only we knew what we know: the transfer of internal knowledge and best practice. New York: The Free Press, 1998.

OLIVEIRA, Fernando. Memory system in organization: an empirical investigation of mechanism for knowledge colletion, storage and access. Journal of Management Studies, Oxford, v. 37, n. 6, p. 811-832, 2000.

PROBST, Gilbert; RAUB, Steffen; ROMHARDT, Kai. Gestão do conhecimento: os elementos construtivos do sucesso. Bookman, 2002.

REÁTEGUI, Ruth. Efetividad de los sistemas de memoria organizacional de uma institución de educación superior. Revista Actualidades Investigativas en Educación, San José, v. 13, n. 1, p. 1-27, jan./abr. 2013.

SHEN, Feng; LIU, Hongli. Modeling university knowledge network. Education Technology and Computer Science, v. 3, p. 132-136, 2010.

SCHEIN, Edgard. What is culture? In: FROST, Peter J. et al. Reframing organizational culture. Newbury: Sage, 1991.

SIMIÃO, Hugo Eduardo. Memória organizacional. 2009. Disponível em:

<http://www.pr.gov.br/celepar/celepar/batebyte/edicoes/2001/bb115/me moria.htm>. Acesso em: 7 fev. 2012.

STEIN, Eric W. Organizational memory: review of concepts and recommendations for management. International Journal of Information Management, Guildford, v. 15, n. 2, p. 17-32, 1995.

STEIN, Eric W.; ZWASS, Vladimir. Actualizing organizational memory with information systems. Informaction Systems Research, Providence, v. 6, n. 2, p. 85-117, 1995.

SVEIBY, Karl Erik. E. A nova riqueza das organizações: gerenciando e avaliando patrimônios do conhecimento. 5. ed. Rio de Janeiro: Campus, 1998.

VON KROGH, Georg; ICHIJO, Kengo; NONAKA, Ikujiro. Facilitando a criação de conhecimento: reinventando a empresa com o poder da inovação. Rio de Janeiro: Campus, 2001.

WALSH, James P.; UNGSON, Gerardo Rivera. Organizational memory. Academy of Management Review, Mississipi, v. 16, n. 1, p. 57-91, 1991. 
Maria Olívia Ferreira Pereira, Helena de Fátima Nunes Silva, José Simão de

Paula Pinto

A memória organizacional nos processos de gestão do conhecimento: um estudo na Universidade Federal do Paraná

WEXLER, Mark N. Organizational memory and intellectual capital. Journal of Intellectual Capital, v. 3, n. 4, p. 393-414, 2002.

WIIG, Karl M. Knowledge management in public administration. Journal of Knowledge Management, v. 6, n. 3, p. 224-239, 2002.

ZAGO, Célia Cristina. Cultura organizacional: dimensões estruturais e aspectos dinâmicos. Informação \& Sociedade: Estudos, João Pessoa, v.15, n. 2, p. 63-93, 2005. Disponível em:

<http://www.informacaoesociedade.ufpb.br/ojs2/index.php/ies/

article/viewFile/32/30>. Acesso em: 3 fev. 2014.

\title{
Title
}

The organizational memory in the process of knowledge management: Study in the Federal University of Paraná

\begin{abstract}
Introduction: Organizational Memory allows the identification, registration, use and reuse of information and knowledge built from practice. The Universities need to improve their practices related to use and reuse of information derived from practical experiences lived and learned.

Objective: Aims to identify Organizational Memory, as one element involving the representation of knowledge and information, as a practice of knowledge management in the Federal University of Paraná.

Methodology: Exploratory study, supported on a literature review, with data collected through an online questionnaire distributed to the staff of the Dean of Research and Post Graduate Studies of Federal University of Paraná.

Results: The results present data on the education of the study personnel. It is observed that employees realize the importance of organizational memory, but not yet aware of their importance as an essential element of management.

Conclusion: Organizational Memory must be used as one component that propitiates to manage the intellectual and individual assets and a tool that should facilitate the sharing and reuse of corporate knowledge.
\end{abstract}

Keywords: Knowledge management. Organizational memory. Knowledge sharing. Knowledge reuse. Organizational culture.

\section{Titulo}

La Memoria Organizacional en los procesos de la gestión del conocimiento: un estudio en la Universidad Federal del Paraná.

\section{Resumen}

Introducción: La Memoria Corporativa permite la identificación, registro, uso y reutilización de información y del conocimiento que son construidos a partir de 
Maria Olívia Ferreira Pereira, Helena de Fátima Nunes Silva, José Simão de

Paula Pinto

A memória organizacional nos processos de gestão do conhecimento: um estudo na Universidade Federal do Paraná

la práctica. Las Universidades requieren mejorar sus prácticas relacionadas al aprovechamiento y reutilización de la información derivada de experiencias vividas y aprendidas por ellas.

Objetivo: Procurar identificar la memoria corporativa como un elemento que implique la representación del conocimiento y de la información como una práctica de la Gestión del Conocimiento en la Universidad Federal de Paraná (UFPR). Metodología: Investigación de tipo exploratoria, apoyada en una revisión de la literatura. La colecta de datos fue realizada por medio de cuestionarios online dirigidos a los funcionarios que laboran en el Departamento de Rectorado de Investigación y Postgrado (PRPPG) de la UFPR.

Resultados: Los resultados presentan datos sobre la educación del personal de estudio. Se observa que los empleados se dan cuenta de la importancia de la memoria de la organización, pero que aún no son conscientes de su importancia como elemento esencial de la administración.

Conclusiones: Se concluye que la memoria corporativa debe ser empleada como un instrumento que propicie el intercambio, interacción y reutilización del conocimiento corporativo e individual.

Palabras clave: Gestión del conocimiento. Memoria organizacional. Intercambio de conocimiento. Reutilización del conocimiento. Cultura organizacional.

Recebido em: 16.05.2014

Aceito em: 31.07.2015

Inf. Inf., Londrina, v. 21, n. 1, p. 348 - 374, jan./abr. 2016.

http:www.uel.br/revistas/informacao/ 